\title{
Variational optimal control technique for the tracking of deformable objects
}

\author{
Nicolas Papadakis and Etienne Mémin \\ IRISA/INRIA Campus de Beaulieu 35042 Rennes Cedex, France \\ npapadak, memindirisa.fr
}

\begin{abstract}
In this paper, a new framework for the tracking of closed curves is described. The proposed approach formalized through an optimal control technique, enables a continuous tracking along an image sequence of a deformable curve. The associated minimization process consists in a forward integration of a dynamical model followed by a backward integration of an adjoint dynamics. This latter pde includes a term related to the discrepancy between the state variables evolution law and discrete noisy measurements of the system. The closed curves are represented through an implicit surface.
\end{abstract}

\section{Introduction}

Tracking the contours of an object is an essential task in many applications of computer vision. Due to the changing shape of deformable or even rigid objects in image sequences such an issue appears to be very challenging in the general case. Another serious difficulty comes from the dimension of the space of closed curves in the general case (infinite in theory). This context makes difficult the use of recursive Bayesian filters such as the particle filter [3], since stochastic sampling in large state spaces is usually completely inefficient. For such an issue, numerous approaches based on the level set representation have been proposed $[5,6,8,12,15,16,19,21]$. All these techniques describe the tracking as successive $2 \mathrm{D}$ segmentation processes sometimes enriched with a motion based propagation step. Segmentation techniques on spatio-temporal data have also been proposed $[1,6]$. Unless the introduction of knowledge on the shape of interest $[10,18]$, these approaches are quite sensitive to noise [13] and exhibit inherent temporal instabilities. Besides, it is difficult in such techniques to require the curve to obey to a specified dynamics and therefore to proceed to a real tracking.

In [22], an approach based on a group action mean shape has been used in a moving average context. Contrary to previous methods, this approach introduces, through the moving average technique, a kind of tracking process. This tracking is restricted to simple motions and does not allow to introduce complex dynamical law defined through differential operators. The explicit introduction of a dynamic law in the curve evolution law has been considered in [15]. However, the proposed technique needs a complex detection mechanism to cope with occlusions. Few works attempted to mix stochastic filtering and a level set representation for curve tracking [7, 20]. As mentioned earlier, these works have to face a high dimensional sampling problem and as a consequence rely on crude discretization of the non linear curve dynamics which may be problematic in some situations. In this paper, we propose a technique related to the optimal control theory $[9,11]$ for the tracking of closed curves. This technique enables to estimate in batch mode the complete trajectory of the level set surface according to a set of noisy measurements and a specified dynamics. This method has the advantage to authorizes naturally to cope with state spaces of high dimension.

\section{Variational tracking}

In this section, we describe the general principles of the technique proposed. This setup relies on control theory recipes $[11,9]$. 
Direct evolution model The state variable representing the feature of interest $X$, is assumed to live in a functional space $\mathcal{W}\left(t_{0}, t_{f}\right)=\left\{X \mid X \in L^{2}\left(t_{0}, t_{f} ; \mathcal{V}\right), \partial_{t} X \in\right.$ $\left.L^{2}\left(t_{0}, t_{f} ; \mathcal{V}\right)\right\}$, where $\mathcal{V}$ is an Hilbert space identified to its dual space. The evolution in time range $\left[t_{0} ; t_{f}\right]$ of the state is described through a (non linear) differential operator $\mathbb{M}: \mathcal{V} \times] t_{0}, t_{f}[\rightarrow \mathcal{V}$, defined up to a control function $\nu \in \mathcal{W}\left(t_{0}, t_{f}\right)$, and an initial value defined up to another control variable $\eta \in \mathcal{V}$ :

$$
\left\{\begin{array}{l}
\left.\partial_{t} X(t)+\mathbb{M}(X(t), t)=\nu(t) \quad \forall t \in\right] t_{0}, t_{f}[, \\
X\left(t_{0}\right)=X_{0}+\eta
\end{array}\right.
$$

We are facing an imperfect dynamical system which depends on the whole trajectory of the control variables, $\nu(t)$, and on the value of a control variable, $\eta$, modeling the uncertainty on the initial condition.

This direct problem (1) will be assumed to be well posed, which means that we first assume that the application $\mathcal{V} \times \mathcal{V} \rightarrow \mathcal{V}: \nu(t), \eta \mapsto X(\nu(t), \eta, t)$ is differentiable and continuous $\forall t \in] t_{0}, t_{f}$ [ and secondly that given $\eta \in \mathcal{V}$, $\nu \in \mathcal{W}\left(t_{0}, t_{f}\right)$ and $t_{f}>t_{0}$, there exists a unique function $X \in \mathcal{W}\left(t_{0}, t_{f}\right)$ solution of problem (1). Let us also assume that some observations $Y \in \mathcal{O}$ of the state variable components are available. These observations may live in a different space (a reduced space for instance) from the state variable. We will nevertheless assume that there exists a (non linear) observation operator $\mathbb{H}: \mathcal{V} \rightarrow \mathcal{O}$, that goes from the variable space to the observation space.

Cost function We aim in that work at estimating the control variable of lower magnitude that minimizes a discrepancy measure between the state variable and the observations. This is expressed by the minimization of a cost function $J: \mathcal{W} \times \mathcal{V} \rightarrow \mathbb{R}$ defined as:

$$
\begin{aligned}
J(\nu, \eta) & =\frac{1}{2} \int_{t_{0}}^{t_{f}}\|Y-\mathbb{H}(X(\nu(t), \eta, t))\|_{R}^{2} d t+\frac{1}{2}\|\eta\|_{B}^{2} \\
& +\frac{1}{2} \int_{t_{0}}^{t_{f}}\|\nu(t)\|_{Q}^{2} d t .
\end{aligned}
$$

Norms $\|\cdot\|_{R},\|\cdot\|_{B}$ and $\|\cdot\|_{Q}$ are induced associated to the scalar products $\left\langle R^{-1} \cdot, \cdot\right\rangle_{\mathcal{O}},\left\langle B^{-1} \cdot, \cdot\right\rangle_{\mathcal{V}}$ and $\left\langle Q^{-1} \cdot, \cdot\right\rangle_{\mathcal{V}}$, where $R, B$ and $Q$ are symmetric positive defined endomorphisms of $\mathcal{V}$. In our application, $R, B$ and $Q$ are respectively called the observation covariance matrix, the initialization covariance matrix and the model covariance matrix.
Differential model In order to compute partial derivative of the cost function with respect to the control variables, system (1) is first differentiated with respect to $(\nu, \eta)$ in the direction $(\delta \nu, \delta \eta)$. Noting $d X=\frac{\partial X}{\partial \nu} \delta \nu+\frac{\partial X}{\partial \eta} \delta \eta \in$ $\mathcal{W}\left(t_{0}, t_{f}\right)$, we obtain the following problem:

$$
\begin{aligned}
& \text { Given }(\nu, \eta) \in(\mathcal{W}, \mathcal{V}), X(t) \text { solution of (1) } \\
& \text { and a perturbation }(\delta \nu, \delta \eta) \in(\mathcal{W} \times \mathcal{V}) \text {, } \\
& d X=\frac{\partial X}{\partial \nu} \delta \nu+\frac{\partial X}{\partial \eta} \delta \eta \in \mathcal{W}\left(t_{0}, t_{f}\right) \text {, is defined such that: } \\
& \left\{\begin{array}{l}
\left.\partial_{t} d X(t)+\left(\partial_{X} \mathbb{M}\right) d X(t)=\delta \nu(t) \quad \forall t \in\right] t_{0}, t_{f}[, \\
d X\left(t_{0}\right)=\delta \eta
\end{array}\right.
\end{aligned}
$$

In this expression, the tangent linear operator $\partial_{X} \mathbb{M}$ is defined as the Gâteaux derivative of the operator $\mathbb{M}$ at point $X$ :

$$
\left(\partial_{X} \mathbb{M}\right) d X(t)=\lim _{\beta \rightarrow 0} \frac{\mathbb{M}(X(t)+\beta d X(t))-\mathbb{M}(X(t))}{\beta} .
$$

The tangent linear operator $\left(\partial_{X} \mathbb{H}\right)$ associated to $\mathbb{H}$ may be defined similarly. The differentiation of cost function (2) with respect to $(\nu, \eta)$ in the direction $(\delta \nu, \delta \eta)$ reads:

$$
\begin{aligned}
\left\langle\frac{\partial J}{\partial \eta}, \delta \eta\right\rangle_{\mathcal{V}} & =-\int_{t_{0}}^{t_{f}}\left\langle Y-\mathbb{H}(X),\left(\partial_{X} \mathbb{H}\right) \frac{\partial X}{\partial \eta} \delta \eta\right\rangle_{R} d t \\
& +\left\langle X\left(t_{0}\right)-X_{0}, \delta \eta\right\rangle_{B} \\
\left\langle\frac{\partial J}{\partial \nu}, \delta \nu\right\rangle_{\mathcal{W}} & =-\int_{t_{0}}^{t_{f}}\left\langle Y-\mathbb{H}(X),\left(\partial_{X} \mathbb{H}\right) \frac{\partial X}{\partial \nu} \delta \nu(t)\right\rangle_{R} d t \\
& +\int_{t_{0}}^{t_{f}}\left\langle\partial_{t} X(t)+\mathbb{M}(X(t)), \delta \nu(t)\right\rangle_{Q} d t .
\end{aligned}
$$

These expressions can be rewritten as:

$$
\begin{aligned}
\left\langle\frac{\partial J}{\partial \eta}, \delta \eta\right\rangle_{\mathcal{V}} & =-\int_{t_{0}}^{t_{f}}\left\langle\left(\partial_{X} \mathbb{H}\right)^{*} R^{-1}(Y-\mathbb{H}(X)), \frac{\partial X}{\partial \eta} \delta \eta\right\rangle_{\mathcal{V}} d t \\
& +\left\langle B^{-1}\left(X\left(t_{0}\right)-X_{0}\right), \delta \eta\right\rangle_{\mathcal{V}} . \\
\left\langle\frac{\partial J}{\partial \nu}, \delta \nu\right\rangle_{\mathcal{W}} & =-\int_{t_{0}}^{t_{f}}\left\langle\left(\partial_{X} \mathbb{H}\right)^{*} R^{-1}(Y-\mathbb{H}(X)), \frac{\partial X}{\partial \nu} \delta \nu(t)\right\rangle_{\mathcal{V}} d t \\
& +\int_{t_{0}}^{t_{f}}\left\langle Q^{-1}\left(\partial_{t} X(t)+\mathbb{M}(X(t)), \delta \nu(t)\right\rangle_{\mathcal{V}} d t\right.
\end{aligned}
$$

where $\left(\partial_{X} \mathbb{H}\right)^{*}$, the adjoint operator of $\left(\partial_{X} \mathbb{H}\right)$, is defined by the scalar product:

$$
\forall X \in \mathcal{V}, \forall Y \in \mathcal{O}\left\langle\left(\partial_{X} \mathbb{H}\right) X, Y\right\rangle_{\mathcal{O}}=\left\langle X,\left(\partial_{X} \mathbb{H}\right)^{*} Y\right\rangle_{\mathcal{V}}
$$


Adjoint evolution model In order to estimate the gradient of the cost function $J$, a first brute force numerical approach consists in computing the functional gradient through finite differences:

$$
\nabla_{e_{k}} J \simeq\left[\frac{J\left(u+\epsilon e_{k}\right)-J(u)}{\epsilon}\right], k=1 \cdots p,
$$

where $u=(\nu, \eta) \in(\mathcal{W}, \mathcal{V}), \epsilon \in \mathbb{R}$ is an infinitesimal perturbation and $\left\{e_{k}, k=1, \ldots, p\right\}$ denotes the unitary basis vectors of the control space $(\mathcal{W}, \mathcal{V})$. Such a computation is impractical for space of large dimension since it requires $p$ integrations of the evolution model for each required value of the gradient functional. Adjoint model technique, as introduced in control theory and data assimilation [11, 9], allows to compute efficiently this gradient functional. To obtain the adjoint equation, the first equation of model (3) is multiplied by an adjoint variable $\lambda \in \mathcal{W}\left(t_{0}, t_{f}\right)$ and integrated on $\left[t_{0}, t_{f}\right]$ :

$$
\begin{aligned}
& \int_{t_{0}}^{t_{f}}\left\langle\partial_{t} d X(t), \lambda(t)\right\rangle_{\mathcal{V}} d t+\int_{t_{0}}^{t_{f}}\left\langle\partial_{X} \mathbb{M} d X(t), \lambda(t)\right\rangle_{\mathcal{V}} d t \\
& =\int_{t_{0}}^{t_{f}}\langle\delta \nu(t), \lambda(t)\rangle_{\mathcal{V}} d t .
\end{aligned}
$$

After an integration by parts of the first term and using the second equation of the differential model (3), we finally get:

$-\int_{t_{0}}^{t_{f}}\left\langle-\partial_{t} \lambda(t)+\left(\partial_{X} \mathbb{M}\right)^{*} \lambda(t), d X(t)\right\rangle_{\mathcal{V}} d t$

$=\left\langle\lambda\left(t_{f}\right), d X\left(t_{f}\right)\right\rangle_{\mathcal{V}}-\left\langle\lambda\left(t_{0}\right), \delta \eta\right\rangle_{\mathcal{V}}-\int_{t_{0}}^{t_{f}}\langle\lambda(t), \delta \nu(t)\rangle_{\mathcal{V}} d t$.

where we introduced the adjoint operator $\left(\partial_{X} \mathbb{M}\right)$ * defined by the scalar product:

$\forall X \in \mathcal{V}, \forall Y \in \mathcal{V} \quad\left\langle\left(\partial_{X} \mathbb{M}\right) X, Y\right\rangle_{\mathcal{V}}=\left\langle X,\left(\partial_{X} \mathbb{M}\right)^{*} Y\right\rangle_{\mathcal{V}}$

In order to obtain an accessible expression for the cost function gradient, we impose to the adjoint variable to be solution of the following adjoint problem:

$\left\{\begin{array}{l}-\partial_{t} \lambda(t)+\left(\partial_{X} \mathbb{M}\right)^{*} \lambda(t)=\left(\partial_{X} \mathbb{H}\right)^{*} R^{-1}(Y-\mathbb{H}(X(t))) \\ \lambda\left(t_{f}\right)=0 .\end{array}\right.$

Functional gradient Combining equations (6), (8) and (10) and recalling that $d X=\frac{\partial X}{\partial \nu} \delta \nu+\frac{\partial X}{\partial \eta} \delta \eta$, the functional gradient is given by:

$$
\begin{aligned}
\left\langle\frac{\partial J}{\partial \nu}, \delta \nu\right\rangle_{\mathcal{W}}+\left\langle\frac{\partial J}{\partial \eta}, \delta \eta\right\rangle_{\mathcal{V}} \\
=\int_{t_{0}}^{t_{f}}\left\langle Q^{-1}\left(\partial_{t} X(t)+\mathbb{M}(X(t)), \delta \nu(t)\right\rangle_{\mathcal{V}} d t-\int_{t_{0}}^{t_{f}}\langle\lambda(t), \delta \nu(t)\rangle_{\mathcal{V}} d t\right. \\
\quad-\left\langle\lambda\left(t_{0}\right), \delta \eta\right\rangle_{\mathcal{V}}+\left\langle B^{-1}\left(X\left(t_{0}\right)-X_{0}\right), \delta \eta\right\rangle_{\mathcal{V}} \\
=\left\langle Q^{-1}\left(\partial_{t} X+\mathbb{M}(X)-\lambda, \delta \nu\right\rangle_{\mathcal{W}}\right. \\
\quad+\left\langle-\lambda\left(t_{0}\right)+B^{-1}\left(X\left(t_{0}\right)-X_{0}\right), \delta \eta\right\rangle_{\mathcal{V}} .
\end{aligned}
$$

The derivatives of the cost function with respect to $\nu$ and $\eta$ are identified as:

$$
\begin{aligned}
& \frac{\partial J}{\partial \nu}=Q^{-1}\left(\partial_{t} X+\mathbb{M}(X)\right)-\lambda \\
& \frac{\partial J}{\partial \eta}=-\lambda\left(t_{0}\right)+B^{-1}\left(X\left(t_{0}\right)-X_{0}\right) .
\end{aligned}
$$

A gradient descent optimization can be set by canceling these components. Introducing $Q$ and $B$, the respective pseudo inverses of $Q^{-1}$ and $B^{-1}$ [2], the state variables update reads:

$$
\left\{\begin{array}{l}
\partial_{t} X(t)+\mathbb{M}(X(t))=Q \lambda(t) \\
X\left(t_{0}\right)-X_{0}=B \lambda\left(t_{0}\right)
\end{array}\right.
$$

The second equation constitutes an incremental update of the initial condition from the value of the adjoint variable at the initial time. This system can be generalized to define the following incremental formulation.

Incremental function Denoting

$$
\left\{\begin{array}{l}
X(t)=\tilde{X}(t)+d X(t) \quad \forall t \in\left[t_{0}, t_{f}\right], \\
\tilde{X}\left(t_{0}\right)=X_{0}
\end{array}\right.
$$

where $\tilde{X}(t)$ is either a fixed component or a previous estimated trajectory of the state variable, equation (13) can be written as:

$$
\begin{array}{rlrl}
\partial_{t} \tilde{X}(t)+\mathbb{M}(\tilde{X}(t)) & =\quad 0 \quad \forall t \in] t_{0}, t_{f}[ \\
\partial_{t} d X(t)+\partial_{\tilde{X}} \mathbb{M}(\tilde{X}(t)) d X(t) & =Q \lambda(t) & \forall t \in] t_{0}, t_{f}[.
\end{array}
$$

Hence, the update of the state variable $X$ is driven by an incremental function $d X$ which depends on the whole trajectory of the adjoint variable $\lambda$. The initial value of this incremental function is given by the second equation of (13):

$$
d X\left(t_{0}\right)=B \lambda\left(t_{0}\right)
$$

Equations (10), (15), (16) and (17) give rise to a data assimilation method with an imperfect dynamical model. A sketch of the whole process is summarized in Algorithm (2.1).

\section{Application to curve tracking}

We will focus in this section on the application of the previous framework to curve tracking. 


\section{Algorithm 2.1 Let $X\left(t_{0}\right)=X_{0}$.}

(i) From $X\left(t_{0}\right)$, compute $\left.X(t), \forall t \in\right] t_{0}, t_{f}[$ with a forward integration of system (15).

(ii) $X(t)$ being given, realize a backward integration of the adjoint variable with the system (10).

(iii) Compute the initial value of the incremental function $d X\left(t_{0}\right)$ with relation (17).

(iv) From $d X\left(t_{0}\right)$, compute $\left.d X(t), \forall t \in\right] t_{0}, t_{f}[$ with a forward integration of system (16).

(v) Update $X=X+d X$.

(vi) Return to (ii) and repeat until convergence

$(J(\nu(t), \eta)<$ threshold $)$.

\subsection{Contour representation and evolution laws}

As we aim at tracking non parametric closed curves that may exhibit topology changes along time, we will rely on an implicit level set representation of the curve of interest $\Gamma(t)$ at time $t \in\left[t_{0}, t_{f}\right]$ of the image sequence [16, 21]. Within that framework, the curve $\Gamma(t)$ enclosing the target to track is implicitly described by the zero level set of a function $\phi(\boldsymbol{x}, t): \Omega \times \mathbb{R}+\rightarrow \mathbb{R}: \Gamma(t)=\{\boldsymbol{x} \in \Omega \mid \phi(\boldsymbol{x}, t)=0\}$.

In order to define a dynamics for the unknown surface, we will assume that the curve is propagated at each frame instant by a given velocity field, $\boldsymbol{w}(\boldsymbol{x}, t)=$ $[u(\boldsymbol{x}, t), v(\boldsymbol{x}, t)]^{T}$, and diffuses according to a mean curvature motion. This dynamics is assumed to be valid up to an additive control function $\nu$ :

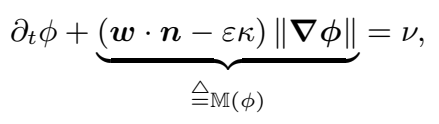

where the curvature and the normal are directly given in term of surface gradient: $\kappa=\operatorname{div}(\boldsymbol{\nabla} \phi /\|\nabla \phi\|)$ and $\boldsymbol{n}=$ $\nabla \phi /\|\nabla \phi\|$. As indicated previously, the motion field transporting the curve is assumed to be given by an external estimator. In practice, we used an efficient and robust version of the Horn and Schunck optical-flow estimator [14]. The additive control function allows us to model inaccuracy of the velocity fields. Since it is rather difficult to infer precise errors model for this dynamics, we fixed the control covariance matrix $Q$ to a constant diagonal matrix (typically
$Q=0.005)$.

Tangent linear evolution operator To apply the setup defined previously, we must first define the expression of the directional derivative of the operators involved. Using equation (18), the evolution operator reads in its complete form:

$$
\mathbb{M}(\phi)=\boldsymbol{\nabla} \phi \cdot \boldsymbol{w}-\varepsilon\|\nabla \phi\| \operatorname{div}\left(\frac{\boldsymbol{\nabla} \phi}{\|\boldsymbol{\nabla} \phi\|}\right) .
$$

This operator can be turned into a more tractable expression for our purpose:

$$
\mathbb{M}(\phi)=\boldsymbol{\nabla} \phi^{T} \boldsymbol{w}-\varepsilon\left(\Delta \phi-\frac{\boldsymbol{\nabla}^{T} \phi \nabla^{2} \phi \boldsymbol{\nabla} \phi}{\|\boldsymbol{\nabla} \phi\|^{2}}\right) .
$$

The corresponding tangent linear operator at point $\phi$ finally reads:

$$
\begin{aligned}
\partial_{\phi} \mathbb{M} \delta \phi & =\boldsymbol{\nabla} \delta \phi^{T} \boldsymbol{w}-\varepsilon\left[\Delta \delta \phi-\frac{\boldsymbol{\nabla} \phi^{T} \nabla^{2} \delta \phi \boldsymbol{\nabla} \phi}{\|\boldsymbol{\nabla} \phi\|^{2}}\right. \\
& \left.+2 \frac{\boldsymbol{\nabla} \phi^{T} \nabla^{2} \phi}{\|\boldsymbol{\nabla} \phi\|^{2}}\left(\frac{\boldsymbol{\nabla} \phi \boldsymbol{\nabla} \phi^{T}}{\|\boldsymbol{\nabla} \phi\|^{2}}-I d\right) \boldsymbol{\nabla} \delta \phi\right] .
\end{aligned}
$$

Operator discretization Before going any further, let us describe the discretization schemes we considered for the evolution law. This concerns the evolution operator, the associated tangent linear operator and the adjoint evolution operator. We will denote as $\phi_{i, j}^{t}$ the value of $\phi$ at image grid point $(i, j)$ at time $t \in\left[t_{0} ; t_{f}\right]$. Using (18) and a semiimplicit discretization scheme, the following discrete evolution model is obtained:

$$
\frac{\phi_{i, j}^{t+\Delta t}-\phi_{i, j}^{t}}{\Delta t}+\mathbb{M}_{\phi_{i, j}^{t}} \phi_{i, j}^{t+\Delta t}=0 .
$$

Considering $\phi_{x}$ and $\phi_{y}$, the horizontal and vertical gradient matrices of $\phi$, the discrete operator $\mathbb{M}$ is obtained as :

$$
\begin{aligned}
& \mathbb{M}_{\phi_{i, j}^{t}} \phi_{i, j}^{t+\Delta t}=\left(\begin{array}{c}
\left(\phi_{x}^{t+\Delta t}\right)_{i, j} \\
\left(\phi_{y}^{t+\Delta t}\right)_{i, j}
\end{array}\right)^{T} \boldsymbol{w} \\
& -\frac{\varepsilon}{\left\|\nabla \phi_{i, j}^{t}\right\|^{2}}\left(\begin{array}{c}
-\left(\phi_{y}^{t}\right)_{i, j} \\
\left(\phi_{x}^{t}\right)_{i, j}
\end{array}\right)^{T} \nabla^{2} \phi_{i, j}^{t+\Delta t}\left(\begin{array}{c}
-\left(\phi_{y}^{t}\right)_{i, j} \\
\left(\phi_{x}^{t}\right)_{i, j}
\end{array}\right),
\end{aligned}
$$

where we used usual finite differences for the Hessian matrix $\nabla^{2} \phi$ and a first order convex scheme [21] for the advective term $\nabla \phi \cdot \boldsymbol{w}$. This scheme enables to compute the surface gradients in the appropriate direction:

$$
\begin{aligned}
\boldsymbol{\nabla} \phi_{i, j}^{T} \boldsymbol{w}_{i, j} & =\max \left(u_{i, j}, 0\right)\left(\phi_{i, j}\right)_{x}^{-}+\min \left(u_{i, j}, 0\right)\left(\phi_{i, j}\right)_{x}^{+} \\
& +\max \left(v_{i, j}, 0\right)\left(\phi_{i, j}\right)_{y}^{-}+\min \left(v_{i, j}, 0\right)\left(\phi_{i, j}\right)_{y}^{+},
\end{aligned}
$$


where $(\phi)_{x}^{-}$and $(\phi)_{y}^{-}$are the left semi-finite differences, whereas $(\phi)_{x}^{+}$and $(\phi)_{y}^{+}$are the right ones. The discrete linear tangent operator is similarly defined as:

$$
\partial_{\phi_{i, j}^{t}} \mathbb{M} \delta \phi_{i, j}^{t+\Delta t}=\mathbb{M}_{\phi_{i, j}^{t}} \delta \phi_{i, j}^{t+\Delta t}-\frac{2 \varepsilon(A B)}{\|\nabla \phi\|^{4}}\left(\begin{array}{c}
\left(\delta \phi_{x}^{t+\Delta t}\right)_{i, j} \\
\left(\delta \phi_{y}^{t+\Delta t}\right)_{i, j}
\end{array}\right)
$$

where $A$ and $B$ are:

$$
\begin{aligned}
& A=\phi_{x}^{t} \phi_{y}^{t}\left(\phi_{x y}^{t} \phi_{x}^{t}-\phi_{x x}^{t} \phi_{y}^{t}\right)+\left(\phi_{y}^{t}\right)^{2}\left(\phi_{y y}^{t} \phi_{x}^{t}-\phi_{x y}^{t} \phi_{y}^{t}\right), \\
& B=\phi_{x}^{t} \phi_{y}^{t}\left(\phi_{x y}^{t} \phi_{y}^{t}-\phi_{y y}^{t} \phi_{x}^{t}\right)+\left(\phi_{x}^{t}\right)^{2}\left(\phi_{x x}^{t} \phi_{y}^{t}-\phi_{x y}^{t} \phi_{x}^{t}\right) .
\end{aligned}
$$

As for the iterative solver involved in the implicit discretization, we used a conjugated gradient optimization. The discretization of the adjoint evolution model is finally obtained as the transposed matrix corresponding to the discretization of the derivative of the evolution law operator.

\subsection{Initial condition}

In order to define an initial condition, we assume that an initial contour of the target object is available. It can be provided by any segmentation technique or specified by the user. In this work we used a simple thresholding technique. Given this initial contour, we initialized the implicit function at the first time as a signed distance function. More precisely, the value of $\phi\left(\boldsymbol{x}, t_{0}\right)$ is set to the distance $g\left(\boldsymbol{x}, \Gamma\left(t_{0}\right)\right)$ of the closest point of the initial curve $\Gamma\left(t_{0}\right)$, with the convention that $g\left(\boldsymbol{x}, t_{0}\right)$ is negative inside the contour, and positive outside. An additive control variable models the uncertainty we have on the initial curve. This initial condition reads:

$$
\phi\left(\boldsymbol{x}, t_{0}\right)=g\left(\boldsymbol{x}, \Gamma\left(t_{0}\right)\right)+\eta(\boldsymbol{x}, t) .
$$

The covariance matrix $B$ associated to the initial state control variable has been defined as a diagonal matrix which fixes a low uncertainty in the vicinity of the initial given curve and an increasing uncertainty as soon as the curve moves away from the initial contour:

$$
B(\boldsymbol{x})=I d-e^{-\left|g\left(\boldsymbol{x}, t_{0}\right)\right|}
$$

\subsection{Measurement equation}

To link the image data to the unknown surface variable we rely on a measurement modeling that involves local probability distributions of the intensity function. This model compares at each point of the image domain a local photometric histogram to two global probability density functions $\rho_{o}$ and $\rho_{b}$ modeling respectively the object and background intensity distribution. These two distributions are assumed to be estimated from the initial location of the target object. The measurements equation we propose reads:

$$
\begin{aligned}
F(\phi, I)(\boldsymbol{x}, t)= & {\left[1-d_{B}\left(\rho_{V_{\boldsymbol{x}}}, \rho_{o}\right)\right]^{2} \mathbf{1}_{\phi(\boldsymbol{x})<0}+} \\
& {\left[1-d_{B}\left(\rho_{V_{\boldsymbol{x}}}, \rho_{b}\right)\right]^{2} \mathbf{1}_{\phi(\boldsymbol{x}) \geq 0}=\epsilon(\boldsymbol{x}, t), }
\end{aligned}
$$

where $d_{B}$ is the Bhattacharya probability density distance measure defined as:

$$
d_{B}\left(\rho_{1}, \rho_{2}\right)=\int_{0}^{255} \sqrt{\rho_{1}(z) \rho_{2}(z)} d z .
$$

and $\rho_{V_{\boldsymbol{x}}}$ is the probability density in the neighborhood of pixel $\boldsymbol{x}$. Let us note that by replacing the densities with intensity average, we retrieve the Chan and Vese functional proposed for image segmentation[4].

The corresponding linear tangent operator in the sense of distributions [4] is:

$$
\partial_{\phi} F=\left(\left[1-d_{B}\left(\rho_{V_{\boldsymbol{x}}}, \rho_{o}\right)\right]^{2}-\left[1-d_{B}\left(\rho_{V_{\boldsymbol{x}}}, \rho_{b}\right)\right]^{2}\right) \boldsymbol{\delta}(\phi)
$$

where $\delta(\cdot)$ is the Dirac function. The covariance associated to the measurements discrepancy has been fixed to a diagonal matrix corresponding to the minimal empiric local photometric covariance:

$R(\boldsymbol{x}, t)=\mathbb{E}\left[M i n\left(\left(1-d_{B}\left(\rho_{V_{\boldsymbol{x}}}, \rho_{o}\right)\right)^{2},\left(1-d_{B}\left(\rho_{V_{\boldsymbol{x}}}, \rho_{b}\right)\right)^{2}\right)\right]$

This measurement equation and the corresponding adjoint linear tangent operator are involved in the adjoint model (10):

$$
-\partial_{t} \lambda(t)+\left(\partial_{\phi} \mathbb{M}\right)^{*} \lambda(t)=\left(\partial_{\phi} F\right)^{*} R^{-1} F(\phi, I)
$$

\section{Experimental results}

As a first example, we tracked a curve delineating alphabetic letters. The measurements consist of a set of four binary letters images, as presented on figure 1. On the same figure, we plotted the results obtained at intermediate instants in order to show how curve deforms continuously 
along the sequence to give some kind of morphing results. Contrary to other results, the curve is here only propagated using its mean curvature motion instead of optical flow. The assimilation principle allows to track the global deformation of the curve along time thanks to the batch approach that considers all the set of available observations.
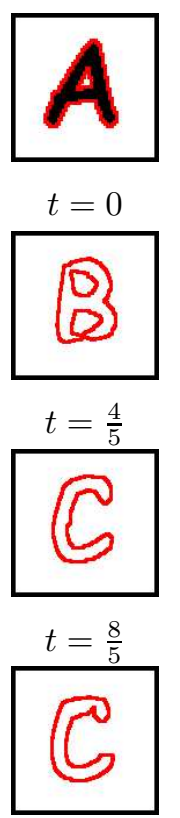

$t=\frac{12}{5}$

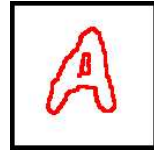

$t=\frac{1}{5}$

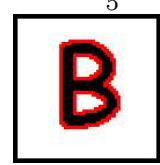

$t=1$

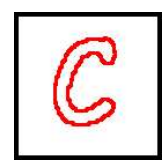

$t=\frac{9}{5}$

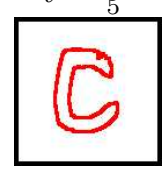

$t=\frac{13}{5}$

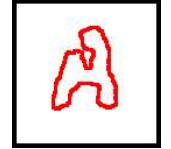

$t=\frac{2}{5}$
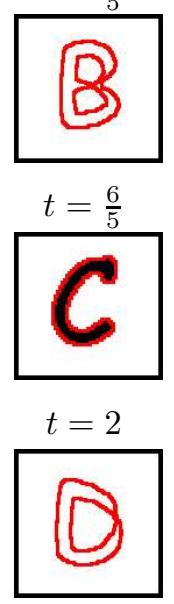

$t=\frac{14}{5}$

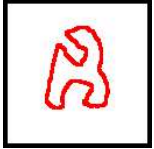

$t=\frac{3}{5}$

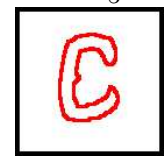

$t=\frac{7}{5}$
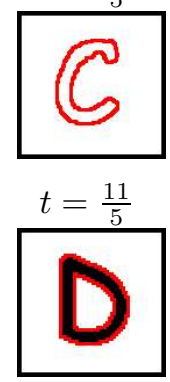

$t=3$
Figure 1. Letter sequence. Result of the assimilation process. The curve is superimposed on the observed letter images at times $t=0,1,2,3$.

We then applied the process to the tracking of a running tiger. This sequence composed of 27 frames is of bad quality: it includes motion blur at some places and is quite noisy. The measurements are provided by local photometric histograms. The initial curve that determines probability density functions of the tiger and the background is obtained with a simple threshold technique. The results shown on figure 2 illustrate the fact that despite the very bad quality of images, the method allows to track the tiger in a consistent way. For this sequence we also plotted on figure 3 a serie of segmentation obtained through a Chan and Vese segmentation process [4] based on the same data model than our measurements model (eq. 21-22) with an additional penalization term on the curve length. As can be observed from figure 3 the mask obtained with this spatial segmentation

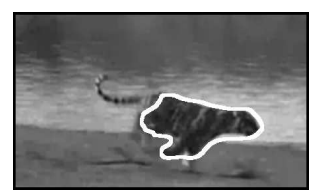

$t=0$

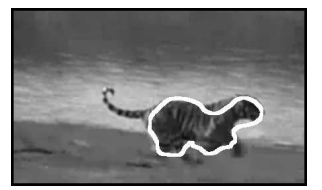

$t=4$

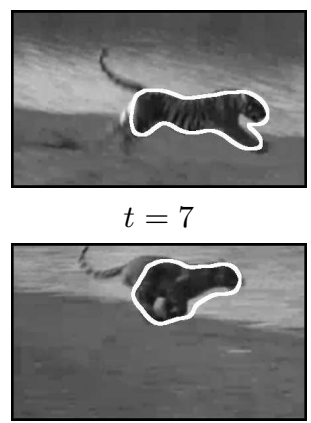

$t=14$

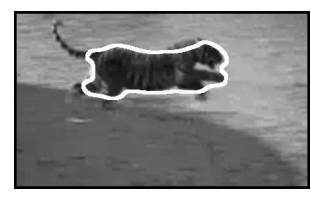

$t=19$

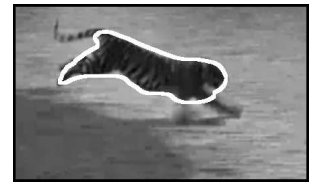

$t=23$

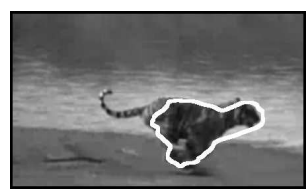

$t=2$

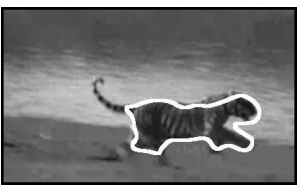

$t=5$

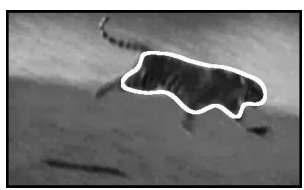

$t=10$

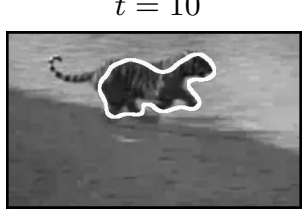

$t=17$

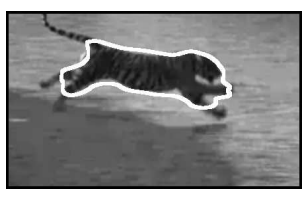

$t=21$

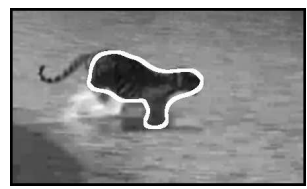

$t=26$
Figure 2. Tiger sequence. Result of the assimilation technique. Despite of the noisy images, the global shape of the tiger is preserved along the time.

technique are of good quality on some images. They nevertheless appears to be unstable along time and would require a delicate tuning of the parameter to obtained a consistent sequence of curves. At the opposite, the curves provided by the proposed technique are more stable in time and consistent with respect to the object shape and its deformation. Compared to traditional segmentation techniques the assimilation techniques provides results which reflect in a more coherent way the topology and the deformation of the target object along time. Due to the bad quality of the image sequence and to the amplitude of the motions we can observe that it is nevertheless difficult for the curve to fit precisely 


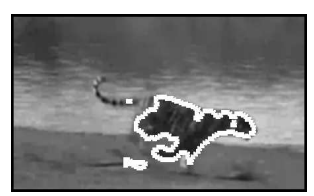

$t=0$

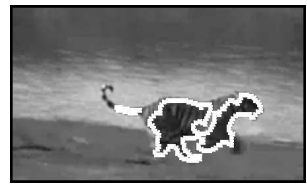

$t=4$

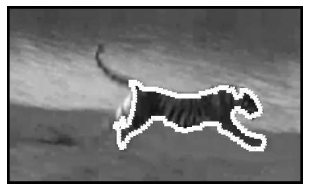

$t=7$

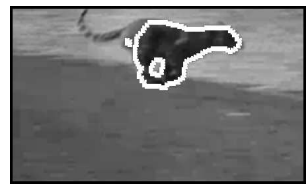

$t=14$

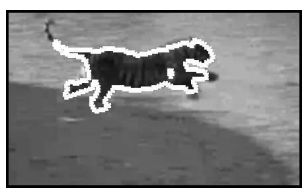

$t=19$

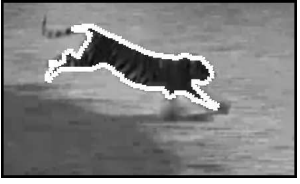

$t=23$

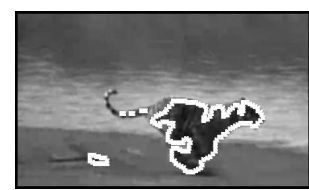

$t=2$

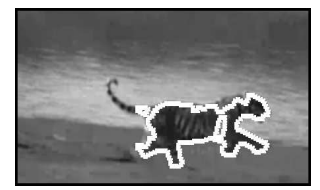

$t=5$

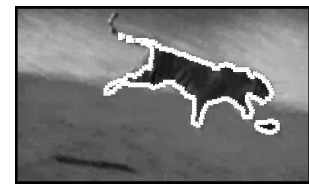

$t=10$

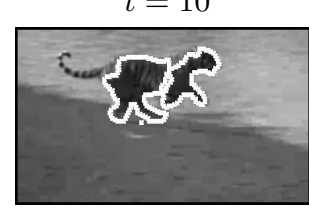

$t=17$

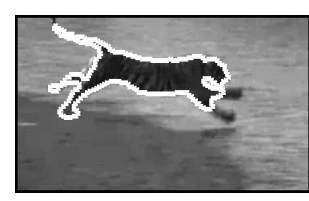

$t=21$

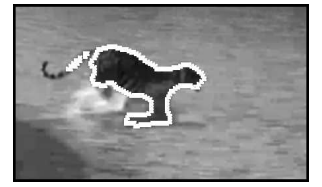

$t=26$
Figure 3. Tiger sequence. Successive segmentations obtained through a Chan and Vese level-set techniques with a data model based on local probability density measurement and a Bhattacharya distance (eq. 21-22).

and in a continuous way to the photometric boundaries of the object.

To further illustrate the differences between the results obtained through assimilation and successive photometric segmentations we finally run our method on a cardiac magnetic resonance imaging sequence ${ }^{1}$. The purpose is here to track the left ventricle. The result of the method are presented on figure 4. As can be observed the target region approximatively delineated in the first image by the user is

\footnotetext{
${ }^{1}$ http://mrel.usc.edu/class/preview.html
}

well tracked. The successive deformations of the region are recovered in a coherent continuous way. The sequence of curve delineates well the evolution of a target region of interest specified at the initialization stage. In comparison, the results obtained from the same initialization with the Chan and Vese techniques show an immediate expansion of the target region to other regions of the image characterized by the same photometric distribution (see figure 5). Incoherent merging or splitting of regions regarding the effective deformations of an object shape is maybe one of the main problems met when running spatio-temporal analysis on the basis of consecutive single spatial analyses (even chained together through their initializations).

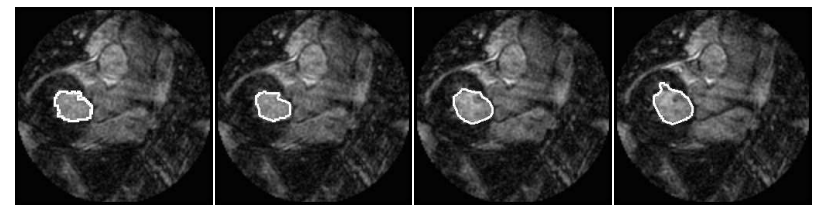

Initialization $t=1$

$t=2$

$t=3$
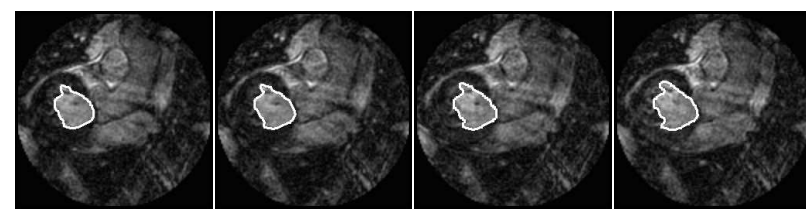

$t=4$

$t=5$

$t=6$

$t=7$

Figure 4. Cardiac magnetic resonance imaging sequence. Result of the assimilation technique with the photometric measurement model based on local probability density. The initial target region is shown in the first image of the top row
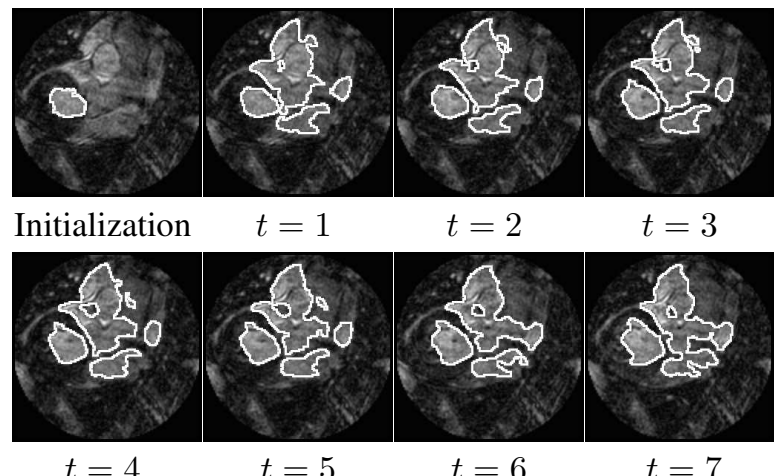

Figure 5. Cardiac magnetic resonance imaging sequence. Successive segmentations obtained through a Chan and Vese level-set techniques with a data model based on local probability density measurement and a Bhattacharya distance (eq. 21-22). The initial target region is shown in the first image of the top row 


\section{Conclusion}

In this paper, a new framework allowing the visual tracking of curves has been presented. The proposed technique relies on data assimilation technique formulated through an optimal control problem [9]. and allows to handle in batch mode the tracking of an implicit surface. The technique is simple and consists in a forward integration of a level-set dynamics coupled with a backward integration of an adjoint dynamics incorporating a data measurement model. The method incorporates only few parameters representing the different errors involved in the considered system. In this work, the curve velocity fields is assumed to be known, nevertheless we could have as well coupled the curve tracking with a similar tracking mechanism on the velocity fields to improve the performances [17]. This will be the subject of future work.

\section{Acknowledgments.}

This work was supported by the European Community through the IST FET Open FLUID project (http://fluid.irisa.fr).

\section{References}

[1] A. Bartesaghi and G. Sapiro. Tracking of moving objects under severe and total occlusions. Int. Conf. on Image Processing (ICIP'05), 1:301-304, 2005.

[2] A. Bennet. Inverse Methods in Physical Oceanography. Cambridge University Press, 1992.

[3] A. Blake and M. Isard. Active contours. Springer-Verlag, London, England, 1998.

[4] T. Chan and L. Vese. Active contours whitout edges. IEEE Trans. Image Processing, 10(2):266-277, 2001.

[5] D. Cremers and S. Soatto. Motion competition: A variational framework for piecewise parametric motion segmentation. Int. J. Comput. Vis., 62(3):249-265, 2005.

[6] R. Goldenberg, R. Kimmel, E. Rivlin, and M. Rudzsky. Fast geodesic active contours. IEEE Trans. on Image Processing, 10(10):1467-1475, 2001.

[7] T. Jiang and C. Tomasi. Level-set curve particles. In Proc. Europ. Conf. Comput. Vis. (ECCV'06), 2006.

[8] R. Kimmel and A. M. Bruckstein. Tracking level sets by level sets: a method for solving the shape from shading problem. Comput. Vis. Image Underst., 62(1):47-58, 1995.
[9] F.-X. Le Dimet and O. Talagrand. Variational algorithms for analysis and assimilation of meteorological observations: theoretical aspects. Tellus, pages 97-110, 1986.

[10] M. Leventon, E. Grimson, and O. Faugeras. Statistical shape influence in geodesic active contours. In Proc. Conf. Comp. Vision Pattern Rec. (CVPR'O0), 2000.

[11] J. Lions. Optimal control of systems governed by PDEs. Springer-Verlag, 1971.

[12] A. Mansouri. Region tracking via level set PDEs without motion computation. IEEE Trans. Pat. Anal. Mach. Intell., 24(7):947-961, 2003.

[13] P. Martin, P. Réfrégier, F. Goudail, and F. Gurault. Influence of the noise model on level set active contours segmentation. IEEE Trans. Pat. Anal. Mach. Intell., 26(6):799-803, 2004.

[14] E. Mémin and P. Pérez. Dense estimation and object-based segmentation of the optical flow with robust techniques. IEEE Trans. Image Processing, 7(5):703-719, 1998.

[15] M. Niethammer and A. Tannenbaum. Dynamic geodesic snakes for visual tracking. In IEEE Conf. on Comp. Vision and Pattern Rec. (CVPR'04), 2004.

[16] S. Osher and J. Sethian. Fronts propagating with curvature dependent speed: Algorithms based on Hamilton-Jacobi formulation. J. of Comput. Physics, 79:12-49, 1988.

[17] N. Papadakis, T. Corpetti, and E. Mémin. Dynamically consistent optical flow estimation. In International Conference on Computer Vision, ICCV'07, October 2007.

[18] N. Paragios. A level set approach for shape-driven segmentation and tracking of the left ventricle. IEEE trans. on Med. Imaging, 22(6), 2003.

[19] N. Paragios and R. Deriche. Geodesic active regions: a new framework to deal with frame partition problems in computer vision. J. of Visual Communication and Image Representation, 13:249-268, 2002.

[20] Y. Rathi, N. Vaswani, A. Tannenbaum, and A. J. Yezzi. Particle filtering for geometric active contours with application to tracking moving and deforming objects. In Proc. Conf. Comp. Vision Pattern Rec. (CVPR'05), 2005.

[21] J. Sethian. Level set methods. Cambridge University-Press, 1996.

[22] S. Soatto, A. Yezzi, and A. Duci. Geometric level sets methods in imaging vision and graphics. Springer Verlag, 2003. 